\title{
Factors that Affect the Success of Search and Rescue Missions : Perceptions of Search and Rescue Crews of Malaysian Police Air Wing
}

\author{
Mohd Harridon ${ }^{1,2}$, Ainan Syabil Shukri ${ }^{1}$ \\ ${ }^{1}$ Aviation Fitness Cluster Universiti Kuala Lumpur Malaysian Institute of Aviation Technology \\ ${ }^{2}$ European Paratroopers \\ DOI: 10.29322/IJSRP.10.11.2020.p10727 \\ http://dx.doi.org/10.29322/IJSRP.10.11.2020.p10727
}

\begin{abstract}
The Malaysian Police Air Wing has a Search and Rescue Unit that actuates Search and Rescue using aircraft and aviation related equipment. The personnel involved are trained professionals with vast experiences. This paper investigated the factors that contributed to the success of Search and Rescue Missions in lieu with aircraft, equipment, experiences, and management. The results were mixed and several initiatives were proposed to mitigate existing predicaments.
\end{abstract}

Index Terms- Aviation Search and Rescue, Search and Rescue Crews, Management Responses

\section{INTRODUCTION}

$\mathrm{I}^{\mathrm{n}}$ Search and Rescue (SAR) missions, several factors contributed to the success of the missions. SAR roles are to search for victims and rescue them. Environments, such as extreme weather, high winds, and others, also played a role in the actuation of the missions. According to Polka, for a SAR mission to be successful, the organization that was responsible for the SAR should be equipped with state of the art equipment and vehicles [1]. Polka also mentioned that a well oiled methodology was also needed to perform seamless SAR operations [1].

Bogue stated that in the light of current situation it is best to consider new technology and equipment for SAR purposes [2]. Bogue even stipulated that it was imperative to gain requirements from stakeholders in order to utilize proper and appropriate equipment for SAR [2]. We found this interesting and important as the research from Bogue had fueled us to actuate our research pertaining to equipment of SAR (this is part of this paper).

Cokorilo had investigated components of SAR and she stated that the type and number of aircraft needed for SAR were gravely important and these acted as factors that decided the success of SAR missions [3]. Cokorilo also went further and had analyzed accident statistics where this had gave an overall view of the SAR ecosystem [3].

We had postulated that in SAR it was important for SAR management to act swiftly and response quickly to alerts in SAR in order to gain successful SAR missions. We had posed this situation to our respondents and their responses were shown in the Results Section of this paper. Braga agreed with us and he stated that in any organizations and industry, it's vital for responses from management be swift and delivered efficiently so that the objectives were met [4]. Braga also iterated that this should be integrated into the culture of most organizations in order to maintain continuity and consistency [4].

For our research, we had used the 4 Point Likert Scale to gain responses from the SAR Crews of the Malaysian Police Air Wing. Willits had pointed out that the usage of Likert Scale was valid and through various literature reviews and analyses Willits concluded that any condemnations of the scale were bogus and invalid [5]. Willits also affirmed that the scale could be used to extract vital information from the respondents or individuals [5].

Sullivan had analyzed in great details the utilization of Likert Scale and she pointed out that the scale was widely used for assessments of workers and was hugely popular in the educational field [6]. Sullivan stipulated that the scale was utilized by various medical organizations that gauged patients satisfactions and others [6]. With this, our approach in the utilization of the Likert Scale was valid and in complete agreement with others in various fields.

\section{LITERATURE REVIEW}

Aviation Search and Rescue is important as it saves lives and contributes to the recoveries of assets and people. Aronica had concluded that SAR is a combination of personnel, tools, vehicles, and others that work in concert to accomplish a mission [7]. Aronica had developed an application that aided the SAR missions by calculating the most appropriate path to be taken to conduct the SAR [7].

Harridon had stipulated that in SAR the most vital entity was the selection of the aircraft to be used where the selection should be based upon performances of the aircraft during SAR missions [8]. This is actually part of Management of SAR Operations where good management would entail appropriate decisions were made to select the best aircraft, equipment, and resources. Harridon further opinionated that proper selection would reduce wastage during SAR operations and would impart significant efficiency throughout the missions [8].

Koester acknowledged that SAR is important and he had produced a new algorithm that conducted SAR in a much efficient manner [9]. With this advent, those involved in SAR were not confronted with wastage in terms of time or resources 
[9]. We applauded this effort and our research dwell upon this matter which was term as "facilities" since "facilities" encompassed physical and non physical entities (such as search algorithm and methodology).

According to the National Search and Rescue Committee (NSARC), it is imperative for agencies involved in SAR to be equipped with aircraft which are suitable for SAR operations and their numbers should also be adequate [10]. NSARC also commented that these aircraft should be in suitable conditions, ready to fly when alerts are received [10].

In a review of SAR operations, Jacobs stated that there were insufficient number of SAR aircraft in the Alaskan Region and this was detrimental to those that resided or worked in that particular region [11]. Jacobs further recommended that aircraft and other SAR equipment be added to bases or agencies that actuated SAR in that region [11]. Jacobs was particularly sympathetic towards workers in the Oil \& Gas industry that worked in perilous environment at the Alaskan Region [11]. We agreed with this issue and we posed this issue to the SAR crews of the Malaysian Police Air Wing and the responses were mixed.

Another issue of SAR was regarding the prompt responses from management when SAR alerts were received. Williams had gone through several cases where he studied responses from management and he stipulated that it was vital that responses from management be quick and without any delay [12]. He was concerned on the confusion and havoc that were created due to

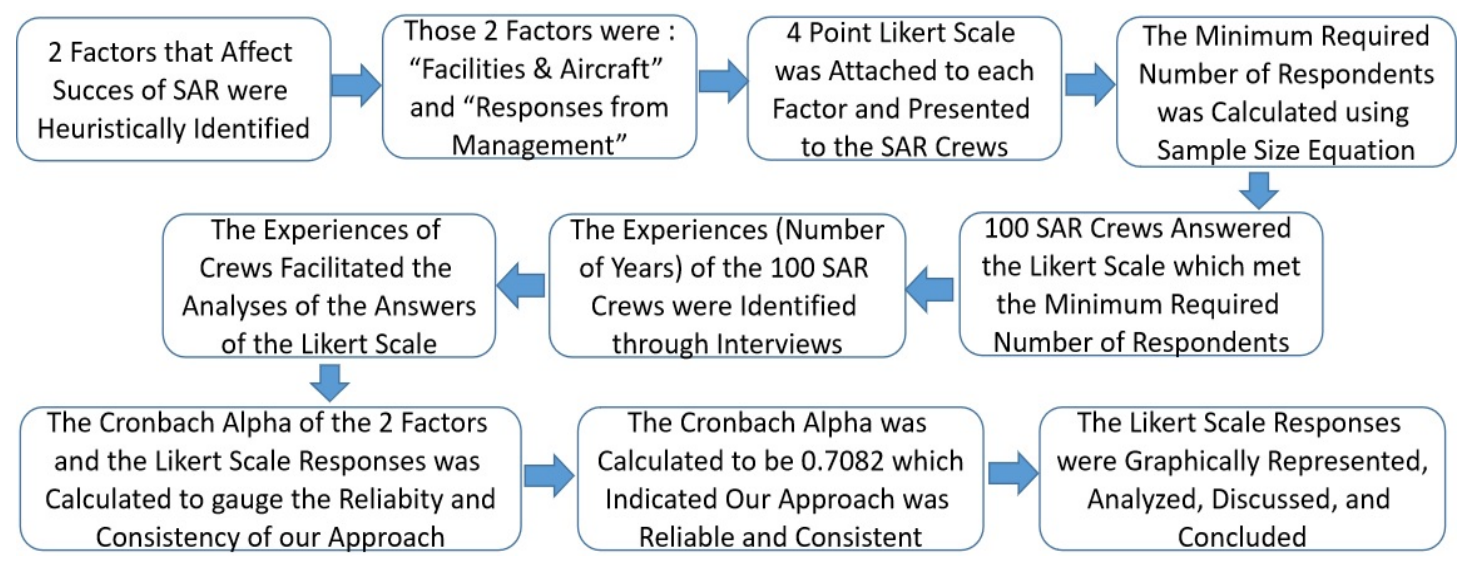

Figure 1. Our Research Methodology

Through heuristic we had identified 2 factors that contributed to the success of missions of SAR. Those 2 factors were "facilities \& aircraft" and "responses from management". Facilities were denoted as physical facilities and non physical facilities (such as search methodology and axiom). These 2 factors were integrated with the 4 Point Likert Scale and we portrayed them to the SAR crews of the Malaysian Police Air Wing.

We had calculated the minimum required number of respondents where the calculation was based upon the Sample Size Equation. The following values were inserted into the equation : population size $=253$, confidence level $=80 \%$, and margin of error $=5 \%$. The population size is the total number of SAR crews at the Malaysian Police Air Wing. The calculation revealed that we needed 100 respondents and we had gained that 100 respondents which met the minimum requirement. We had also interviewed the SAR crews with regards to their experiences. non responsiveness from management [12]. We portrayed this issue to the crews of Malaysian Police Air Wing and the portrayal was via 4 Point Likert Scale.

Jia had used the Likert Scale to compare cultures from different countries [13]. Jia had collected data of students from universities across 16 countries and the collection was via Likert Scale [13]. Jia stated that the Likert Scale was able to perform the comparison and was satisfied with the outcome [13].

Chimi explained that the utilization of Likert Scale had provided advantages in terms of comprehensive analyses [14] Chimi also stated that the scale had the ability to capture hidden meanings and topics which were sensitive were easily discerned by the scale [14].

Janda had also used Likert Scale for her research pertaining to native language [15]. She had applied the scale to measure the nuances of the native speakers and had given the speakers several verbs in order to capture the responses from the speakers [15]. We can evidently observed that the application of Likert Scale was myriad in numerous different circumstances and this somehow validated our usage of the scale in capturing the responses of the SAR crews of the Malaysian Police Air Wing.

\section{Methodology}

Figure 1 showed the methodology of our research.

\section{was Attached to each \\ Factor and Presented} umber of Respondents was Calculated using Sample Size Equation Concluded
Their experiences were required as these were used to aid our analyses of the responses from the Likert Scale. We went further where we had calculated the Cronbach Alpha of our responses and factors and we had gained a value of 0.7082 . This showed that our method was acceptable, consistent, and had sufficient reliability. It had to be noted that the responses which consisted of "strongly agree", "agree", "disagree", and "strongly disagree" were denoted as $1,2,3$, and 4 respectively in order to calculate the Cronbach Alpha value.

The responses were represented in graphical forms and analyzed. Discussions and conclusions were actuated based upon the responses and also were cross checked against the experiences of the SAR personnel. This gave credence to our analyses, discussion, and conclusion. 
IV. RESULTS

The results are shown in Figures 2, 3, 4, 5, and 6.

\section{Experiences in Search \& Rescue}

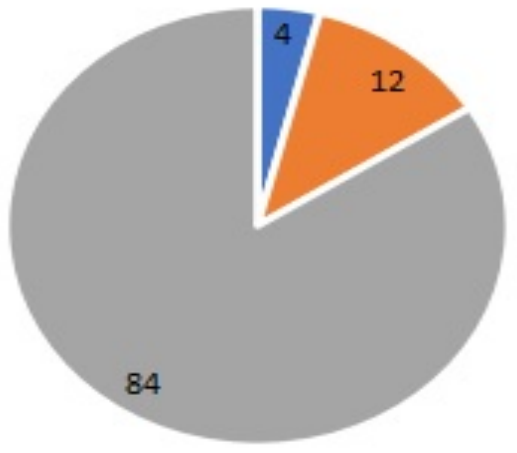

- 1-5years | 6-10years = More than 10 years

Figure 2. Experiences of Crews

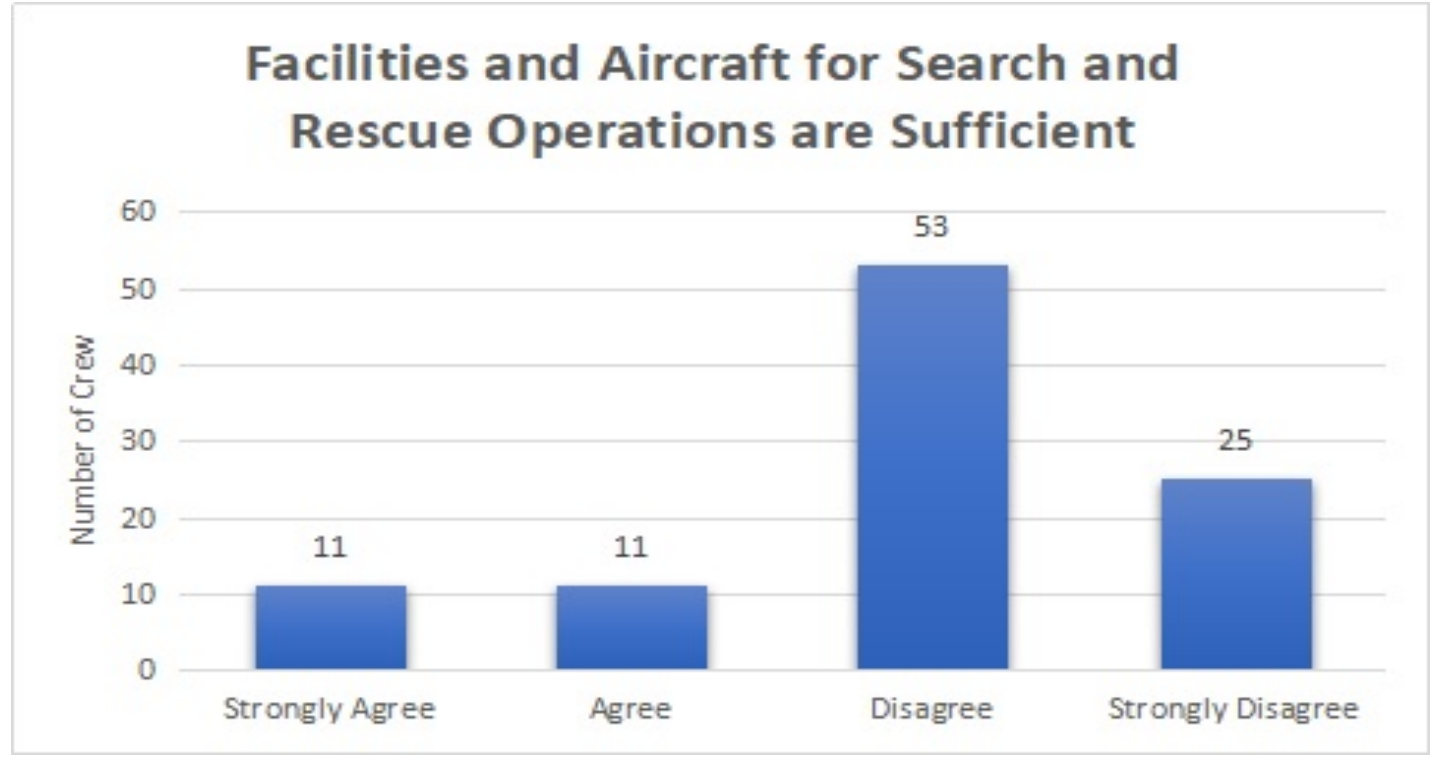

Figure 3. Facilities and Aircraft 


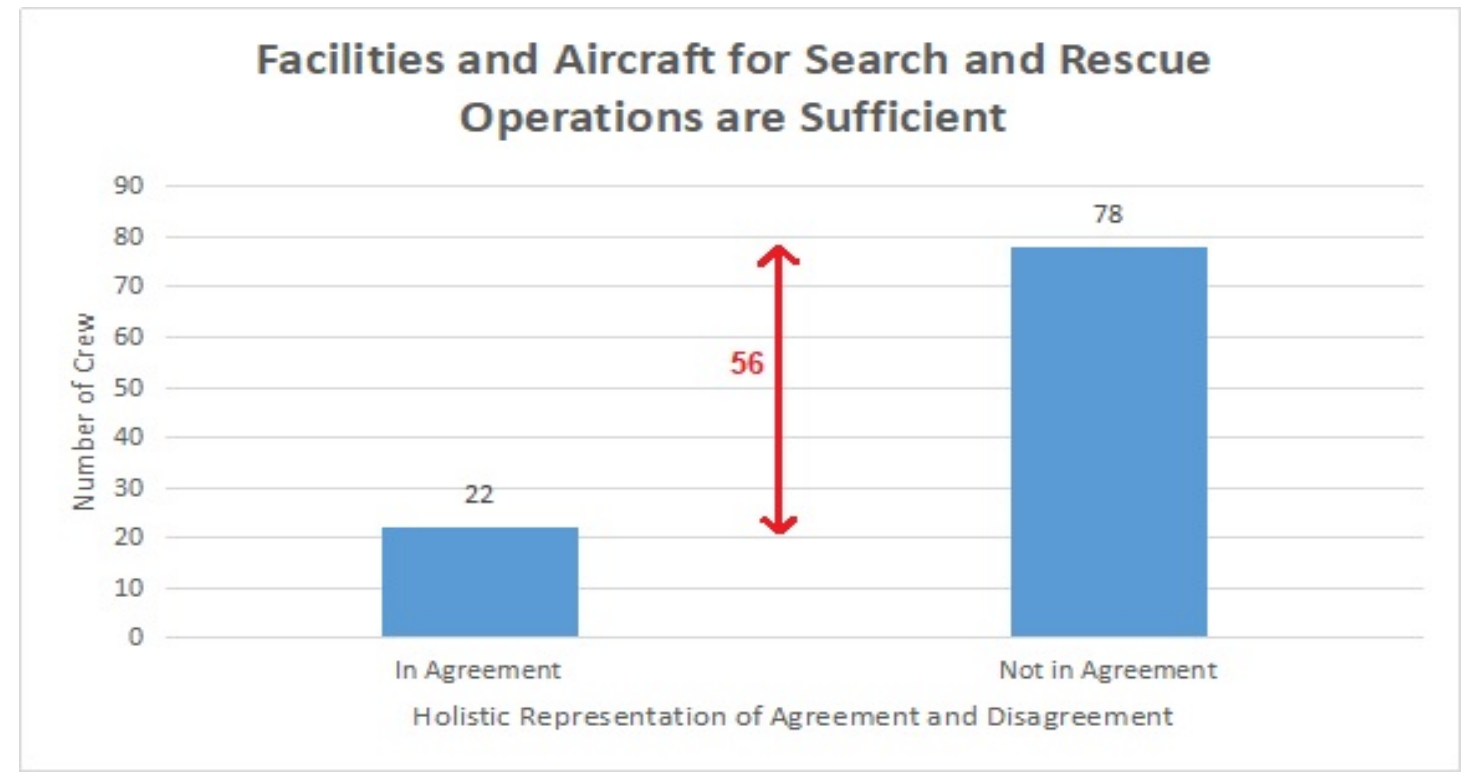

Figure 4. Agreement \& Disagreement - Facilities \& Aircraft

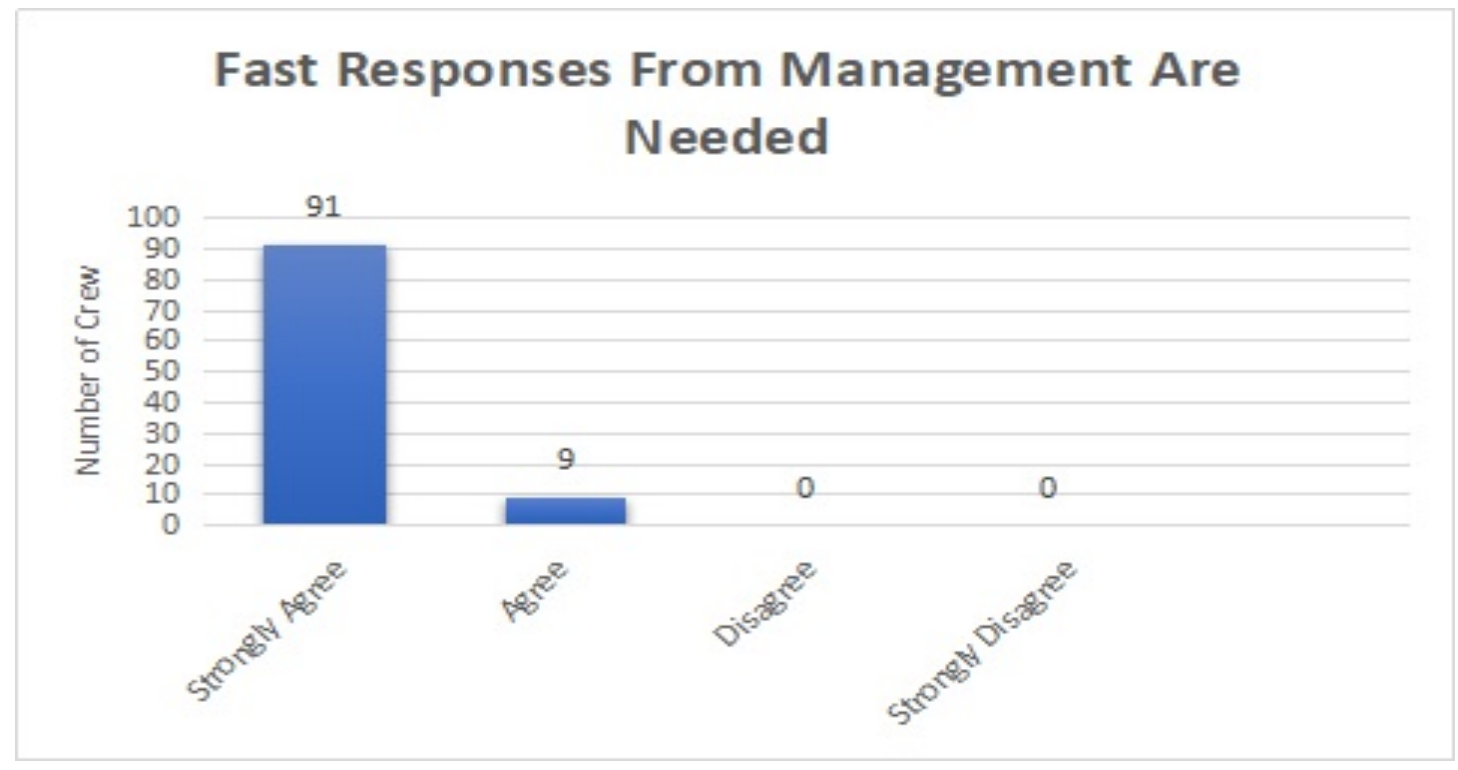

Figure 5. Responses From Management 


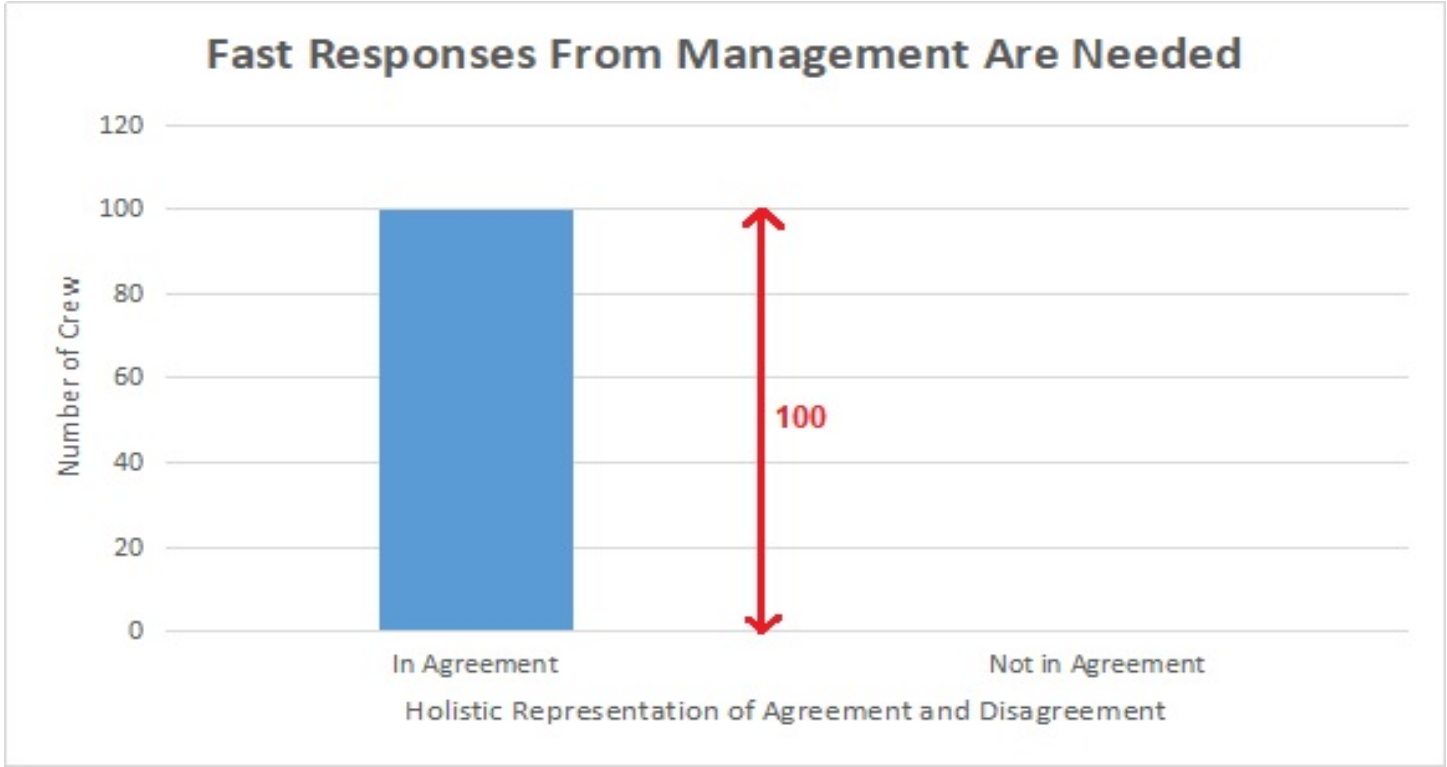

Figure 6. Agreement and Disagreement - Responses from Management

\section{DISCUSSION}

Peering at Figure 2, it can be seen that majority of the SAR crews $(84 \%)$ had more than 10 years experiences. This was an exceptionally good figure as the SAR operations were performed by individuals that were well versed in SAR and had the fortitude to handle emergencies that existed. Only $4 \%$ of the crews had experiences between 1 till 5 years. This seems to be a concern but we managed to get hold of information which indicated that new crews were properly trained before they were allowed to be part of the SAR operations. Also experiences here also mean the ability of the SAR crews to use appropriate methods and tools to maintain their physical fitness. Crews that had enormous experiences were able to identify the appropriate method to enhance their fitness level. Harridon had studied the realm of physical fitness in the field of Aviation and results of the study had shown that high level of physical fitness is important in maintaining safety and delivering outputs [16]. Harridon also stated that physical fitness is a continuous process that should be ingrained in one's culture [16].

In Figure 3, the crews were posed with the statement "the facilities and aircraft for SAR missions were adequate". A great number had disagreed (53\%) and $25 \%$ of the crews had strongly disagreed. Only $11 \%$ agreed and $11 \%$ strongly agreed. This was a grave issue where the majority had stated that the facilities and aircraft were not in sufficient manner in terms of numbers and appropriateness. This should be taken seriously as majority of the crews with more than 10 years experiences had deemed it so. Those with extensive experiences would know best, hence we proposed a review upon the budgetary constraints of the organization in order to acquire more aircraft and improve current facilities.

Figure 4 showed the holistic representation of "agreement" and "disagreement" pursuant to the issue of facilities and aircraft of SAR operations. $78 \%$ of the crews stipulated that the facilities and aircraft were not adequate while $22 \%$ stated otherwise. This $22 \%$ might had not been attached to numerous SAR missions and thus could not offer a comprehensive view of the situation. But we cautioned that more questionings should be in placed to extract reasons of their stipulations with regards to the adequacy of facilities and aircraft.

In figure 5 we had posed an issue regarding responses from management when SAR alerts were received. $91 \%$ of the crews strongly agreed that swift and quick responses from management were needed in order to increase the chances of finding the victims and saving lives. $9 \%$ of the crews also agreed with this notion. There were no disagreements regarding this. It had to be stated that SAR crews of different degrees and levels of experiences were in unison for responses be actuated in a prompt and quick manner. Its plausible that all crews were indoctrinated to act swiftly during emergencies where this indoctrination was done during basic SAR training.

In Figure 6, which showed the holistic representation of agreement and disagreement, it can be observed that $100 \%$ of the SAR crews were in agreement that fast responses were needed from management. It would be best if those in management were integrated with the hands-on crew so that they could produce decisions on the spot during SAR alerts. This means those in management were also required to be on call in order to facilitate the decision making processes.

\section{CONCLUSIONS}

We had gauged the responses of the SAR crews of the Malaysian Police Air Wing. Factors that affected the success of SAR missions were aircraft, facilities, and responses from management. Data and analyses indicated that facilities and aircraft had to be in appropriate manner in terms of numbers and adequacy in order for SAR sorties to be accomplished successfully and efficiently. Responses of SAR management were also vital and had to be prompt in order to actuate actions early so that victims could be located in a timely manner and lives could be saved. 


\section{REFERENCES}

[1] Polka, M., "The Use of UAV's for Search and Rescue Operations", Transcom 2017 : International Scientific Conference on Sustainable, Modern and Safe Transport, Procedia Engineering 192 (2017) 748 - 752, DOI : 10.1016/j.proeng.2017.06.129.

[2] Bogue, R., "Disaster Relief, and Search and Rescue Robots : The Way Forward", Industrial Robot, 2019, Volume 46, No. 2, Pages 181 - 187, DOI 10.1108/IR-11-2018-0227.

[3] Cokorilo, O., "Aviation Safety Risks in Maritime Search and Rescue (SAR) Operations", 5th International Maritime Science Conference, IMSC 2013, Split Croatia, April 2013.

[4] Braga, C.I., "Improving the Organizational Performance in Automotive Manufacturing by Using Fast Response on Layers at Quality Issues", Applied Mechanics and Materials, Volume 384, Pages 211 - 216, ISSN 1662 - 7482, DOI : 10.4028 / www.scientific.net / AMM.834.211.

[5] Willits, F.K., "Another Look at Likert Scales", Journal of Rural Social Sciences, Volume 31, Issue 3, 2016, Pages 126 - 139, Southern Rural Sociological Association.

[6] Sullivan, G.M., "Analyzing and Interpreting Data from Likert-Type Scales", Journal of Graduate Medical Education, December 2013, Volume 5, Issue 4 , Pages 541 - 542, PMID : 24454995, DOI : 10.4300/jgme-5-4-18.

[7] Aronica, S., "An Agent-Based System for Maritime Search and Rescue Operations", Central Europe (CEUR) Workshop Proceedings, Volume 621, Paper 21, ISSN 1613-0073.

[8] Harridon, M., "Perception of Search and Rescue Crew of Malaysian Police Air Wing towards General Issues of Search and Rescue", International Journal of Scientific and Research Publications, Volume 10, Issue 10, October 2020, ISSN 2250-3153, DOI : 10.29322/IJSRP.10.10.2020.p10630

[9] Koester, R.J., "Use of the Visual Range of Detection to Estimate Effective Sweep Width for Land Search and Rescue Based on 10 Detection Experiments in North America", Wilderness Environ Med, 2014 June, 25(2), Pages 132 - 142, DOI : 10.1016 / j.wem.2013.09.016.

[10] National Search and Rescue Committee, "United States National Search and Rescue Supplement to the International Aeronautical and Maritime
Search and Rescue Manual", The Homeland Security Digital Library, Washington DC, May 2000.

[11] Jacobs, J., "Search and Rescue Operational Support and Limitations", National Petroleum Council Study on Research to Facilitate Prudent Artic Development, August 8, 2014, National Petroleum Council Washington DC.

[12] Williams, T.A., "Organizational Response to Adversity : Fusing Crisis Management and Resilience Research Streams", Academy of Management Annals, Volume 11, No 2, 1st March 2017, DOI : 10.5465/annals.2015.0134.

[13] Jia, H., "On Enhancing the Cross-Cultural Comparability of Likert Scale Personality and Value Measures : A Comparison of Common Procedures", European Journal of Personality, Volume 31, Issue 6, November December 2017, Pages 642 - 657, DOI : 10.1002/per.2132.

[14] Chimi, C.J., "The Likert Scale : A Proposal for Improvement Using QuasiContinuous Variables", Proc ISECON 2009, v26 (Washington DC), EDSIG, Nov 8, 2009.

[15] Janda, L.A., "Five Statistical Models for Likert-Type Experimental Data on Acceptability Judgments", Journal of Research Design and Statistics in Linguistics and Communication Science, Volume 3, No. 2, 2016, DOI : $10.1558 /$ jrds.30822.

[16] Harridon, M., "Rate of Perceived Exertion of Potential Avionics Male Students and Proposed Exercises to Decrease Strain", International Journal of Engineering \& Technology, 7 (4.36) (2018) 374-378, DOI : 10.14419/ijet.v7i4.36.28145

\section{AUTHORS}

First Author - Mohd Harridon, Aviation Fitness Cluster Universiti Kuala Lumpur Malaysian Institute of Aviation Technology,mdharridon@unikl.edu.my

Second Author - Ainan Syabil Shukri, Aviation Fitness Cluster Universiti Kuala Lumpur Malaysian Institute of Aviation Technology 